\title{
Incidence of Pseudophakic Cystoid Macular Oedema Post-Cataract Surgery in Illawarra Shoalhaven Local Health District, Australia
}

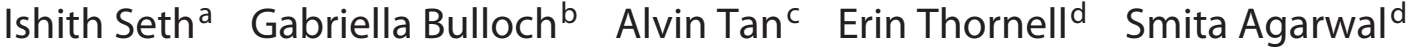 \\ alllawarra Shoalhaven Local Health District, Wollongong Hospital, Wollongong, NSW, Australia; baculty of Science, \\ Medicine and Health, University of Melbourne, Melbourne, VIC, Australia; 'Sydney Local Health District, Royal Prince \\ Alfred Hospital, Camperdown, NSW, Australia; ${ }^{d}$ Wollongong Eye Specialists, Wollongong, NSW, Australia
}

\section{Keywords}

Pseudophakic cystoid macular oedema · Cataract ·

Comorbidities

\section{Abstract}

Background: Post-operatively, cataract surgery is associated with pseudophakic cystoid macular oedema (PCMO) causing vision disturbances. The presence of comorbidities may increase the incidence of PCMO post-cataract surgery. Objective: This observational study aimed to assess the incidence of PCMO in Australia (Illawarra region) and identify risk factors for developing PCMO. Methods: Retrospective analysis was performed on data from patients who underwent uncomplicated phacoemulsification and intraocular lens implantation between 1st March and June 30, 2016. Demographics, comorbidities, central subfield thickness (CST), visual acuity, and intraocular pressure (IOP) were collected preoperatively, day 1 , and weeks 2,4 , and 6 post-operatively. Statistical analysis was performed using SPSS v.27.0 and GraphPad Prism v.9.0. The median and 95\% confidence intervals were used to describe data. Logistic regression and $x^{2}$ tests were used to describe the associations. We followed the Declaration of Helsinki guidelines. Results: Fifty right and 35 left cataract eyes were operated on $(58.8 \%$ were females; average age $72.8 \pm 8.146$ years). Total PCMO incidence was $10.6 \%$, and true PCMO incidence (removing PCMO risk factors) was $4.2 \%$ at week 6 post-operatively. CST was slightly increased between pre- and post-cataract surgery at week $4(p=0.002)$ and week $6(p<0.0001$; median $=$ $259 \mu \mathrm{m}, 264 \mu \mathrm{m}$, and $263 \mu \mathrm{m}$, respectively). IOP was found to be decreased $(p<0.0001)$ compared to day 1 (median $=$ $17 \mathrm{~mm} \mathrm{Hg}$ ) and week 6 (median $=13 \mathrm{~mm} \mathrm{Hg}$ ). The probability of developing PCMO (odds ratio $[\mathrm{OR}]=3$ ) and vitreomacular traction $(O R=2.9)$ was higher in diabetic patients compared to non-diabetic patients and in patients $>65$ years old $(O R=1.5)$. Conclusion: The true incidence of PCMO was found to be the greatest at 2-4 weeks post-operatively. Patients with diabetes or advanced age ( $>65$ years) are at an elevated risk of developing PCMO after cataract surgery. The treatment regimens for the comorbid populations, especially diabetic patients, remain limited, and future efforts should target pharmaceutical management for these groups.

(C) 2022 The Author(s)

Published by S. Karger AG, Basel karger@karger.com www.karger.com/bmh

Karger $\stackrel{\text { ' }}{5}$

GOPEN ACCESS
(C) 2022 The Author(s)

Published by S. Karger AG, Basel

This is an Open Access article licensed under the Creative Commons Attribution-NonCommercial-4.0 International License (CC BY-NC) (http://www.karger.com/Services/OpenAccessLicense), applicable to the online version of the article only. Usage and distribution for commercial purposes requires written permission.
Correspondence to:

Smita Agarwal, smitaagarwal@ hotmail.com 


\section{Introduction}

Cataract is an opacification or change in lens homogeneity within the eye involving the anterior epithelium, capsule, cortex, or nucleus [1]. It accounts for $50 \%$ of blindness in middle- and low-income countries but is present in only $5 \%$ of the population in developed countries [2]. Currently, treatment involves removing the cataract through small-incision phacoemulsification and implanting a synthetic intraocular lens (IOL) [3].

Pseudophakic cystoid macular oedema (PCMO) is a post-operative complication of cataract surgery that is characterized by cystic spaces and symptomatic vision loss (6/12 or worse). The incidence of PCMO after cataract surgery (1-2 months) has been reported to be about 20-30\% [4] Irvine defined PCMO in 1953 to describe cases of mysterious vision loss post-cataract surgery [5]. It is the most common post-operative complication causing impaired vision following cataract surgery and resolves spontaneously in most people but persists in others, resulting in permanent vision disturbances [6]. The pathogenesis of PCMO is thought to involve the release of pro-inflammatory mediators such as prostaglandins and leukotrienes, light toxicity, and mechanical irritation of perifoveal retinal capillaries and irido-vitreal traction [7]. Inflammatory mediators disrupt the blood-aqueous and blood-retinal barriers, increasing vascular permeability and oedema $[8,9]$.

In 1998, the incidence of angiographic PCMO was $30 \%$ [10], but surgical techniques and improved drug therapy have reduced PCMO incidence to $0.1 \%-3.8 \%$ of cases [11]. Its prevalence peaks between 4 and 6 weeks post-operatively [12], and full duration ranges between 72 and 249 days [13]. In most people, it recovers spontaneously within 6 months [14].

Risk factors associated with PCMO include posterior capsule rupture, type 2 diabetes mellitus (DM), primary open-angle glaucoma (POAG), and pre-existing epiretinal membrane [12]. Schimier et al. [15] showed a significant increase in PCMO incidence in diabetic patients compared to non-diabetic (3.05\% vs. $1.73 \%)$. DM patients were also recently estimated to have a 4 -fold risk of developing PCMO compared to healthy subjects [16].

Although the associations between cataract surgery and PCMO have been discussed in many articles, the incidence and time course vary $[17,18]$. In this study, a retrospective analysis was performed on patients undergoing routine cataract surgery to report the incidence and risk factors for PCMO in the Illawarra Shoalhaven local health district (ISLHD) of Australia and associated changes in visual acuity.

\section{Methods}

Eighty-five consecutive patients who underwent uncomplicated unilateral phacoemulsification and monofocal IOL insertion by a single ophthalmologist in a regional public hospital between March 1, 2016, and June 30, 2016, were considered for inclusion in the study. Patients were excluded if they had corneal, retinal, or macular pathology that may compromise visual outcomes. Patients were also excluded if they experienced intra- or post-operative complications. Patients presenting with cystoid macular oedema preoperatively were also excluded.

\section{Surgical Technique}

All surgeries were performed by 1 surgeon (S.A.). Aspherical hydrophobic AcrySof ${ }^{\circledR}$ IQ monofocal lenses (SN60WF, Alcon) were selected using target refraction of $0.0 \pm 0.25 \mathrm{D}$. Toric lenses were used only for patients with preoperative astigmatism of $\geq 1.5$ $\mathrm{D}$ due to the public health system financial constraints. The surgery was performed either under local anaesthesia using topical $0.5 \%$ proxymetacaine hydrochloride drops or a $2 \%$ lignocaine subtenon block or under general anaesthesia as per patient and anaesthesiologist election [19]. For phacoemulsification, 2-side ports were made using a 1-mm ophthalmic knife (MSL10, MANI ${ }^{\circledR}$ Inc.), viscoelastic was injected, and a $2.3-\mathrm{mm}$ main incision was made at $180^{\circ}$ using a $2.3-\mathrm{mm}$ ophthalmic knife (MSL23, MANI ${ }^{\circledR}$ Inc.). A continuous curvilinear capsulorrhexis measuring approximately 5 $\mathrm{mm}$ was performed using a $0.5 \times 16-\mathrm{mm}$ cystotome $(581610$, Beaver-Visitec International). After hydrodissection, phacoemulsification of the nucleus and aspiration of the cortex were performed [19]. The IOL was inserted into the bag, viscoelastic was aspirated, and antibiotic ( $1 \mathrm{mg} / \mathrm{mL}$ cephazolin) was injected intracamerally. Wounds were hydrated and checked for leaks, and subconjunctival injection of approximately $0.2 \mathrm{~mL}$ of dexamethasone $(4 \mathrm{mg} /$ $\mathrm{mL}$ ) was administered [19].

\section{Post-Operative Care}

Following surgery, patients were advised to use ciprofloxacin $(0.3 \% \mathrm{w} / \mathrm{v})$ eye drops for $2-3$ weeks, diclofenac sodium $(0.1 \% \mathrm{w} / \mathrm{v})$ eye drops for 10 days, and phenylephrine hydrochloride ( $1 \% \mathrm{w} / \mathrm{v}) /$ prednisolone acetate $(0.12 \% \mathrm{w} / \mathrm{v})$ eye drops for 4 weeks 4 times daily post-operatively.

\section{Clinical Examination}

Eligible patients were recruited on day 1 post-operatively following uncomplicated unilateral cataract surgery and provided informed consent prior to participation. A routine examination was conducted on this day including slit-lamp examination to assess post-operative inflammation and visual acuity by optometrists. At weeks 2,4 , and 6 post-operatively, the following examinations were conducted: slit-lamp examination, best-corrected visual acuity (BCVA), and distance visual acuity (UDVA) using a reading chart at $40 \mathrm{~cm}$ and a Snellen chart at $6 \mathrm{~m}$, respectively. Refraction was measured by an optometrist using an autorefractor and manifest refraction with trial frames, and intraocular pressure (IOP) was measured using Goldmann tonometry (Tono-Pen ${ }^{\circledR}$, Reichert Technologies, Erie, NY, USA). Retinal changes were monitored by an optometrist 1 week preoperatively and post-operatively at weeks 2, 4, and 6 using fundus examination and a retinal optical coherence tomography (OCT) macular cube $512 \times 128$ scan of the macular and optic-nerve heads (Cirrus HD, Zeiss) to identify mac- 
ular thickening and cystic spaces indicative of PCMO (Fig. 1). Vitreomacular traction (VMT) was screened pre- and at weeks 2, 4, and 6 post-operatively, aligned with the International VMT OCT classification system, vitreous detachment indicated by elevation of cortical vitreous above the retinal surface (focal width of attachment $\leq 1,500 \mu \mathrm{m}$ and broad width of attachment $>1,500 \mu \mathrm{m}$ ) in the perifoveal area in OCT was diagnosed as VMT [20]. Central corneal thickness was measured using ultrasound pachymetry (Pachmate, DGH Technology Inc., Chester, PA, USA). Cataracts (density and type), age-related macular degeneration (AMD), anterior chamber depth (ACD), and corneal haze were screened and diagnosed preoperatively by an experienced ophthalmologist (S.A.) with use of relevant equipment including computerized densitometry on a lamp with a slit beam at $30-45^{\circ}$ angle to the cataract (Zeiss Scheimpflug SL 220, OCT; CIRRUS HD, Zeiss; and Good-Lite, Amsler Grid Chart set, Expanded 730026). The diagnosis of eye pathologies and management were aligned with the ISLHD guidelines, and visual acuity worse than $20 / 40-20 / 50$ or symptomatic decreased vision was a prerequisite for cataract surgery. Full dilated fundus examination was done between 4 and 6 weeks postcataract surgery. A diagnosis of PCMO was made if post-operative CST was greater than $295 \mu \mathrm{m}$, and perifoveal cysts and localized foveal oedema were present on OCT.

The protocol for this study was designed in accordance with the Declaration of Helsinki. This study protocol was reviewed and approved by the University of Wollongong/ISLHD Health and Medical Human Research Ethics Committee, approval number $2016 / 008$. Informed written consent was obtained from participants (or their parent/legal guardian/next of kin) to participate in the study.

\section{Statistical Analysis}

Demographic data were collected including sex, age, eye laterality, and comorbidities such as DM, POAG, AMD, ACD, dense cataract, and corneal haze. Our primary outcomes were incidence of PCMO and VMT pre- and post-cataract surgery and the incidence of PCMO post-cataract surgery with predisposing risk factors including DM, advanced age ( $>65$ years), and VMT. Our secondary outcomes were visual acuity changes post-cataract surgery, IOP, BCVA, and an association between ACD, dense cataract, and corneal haze with poor visual acuity.

Statistical analysis and data visualization were performed using IBM SPSS (IBM Corp., released in 2020, IBM SPSS Statistics for Windows, version 27.0., Armonk, NY, USA) and GraphPad Prism v.9 (GraphPad Software, San Diego, CA, USA). Outcomes at weeks 2, 4, and 6 were compared to baseline (preoperative data for CST and IOP values, day-1 post-operative data for pachymetry, UDVA values, and week- 2 data post-operative for BCVA value). The Shapiro-Wilk test was used to test normality; if $p>0.05$, data are considered normal. For numeric outcomes, the difference between normally distributed data was analysed using a paired sample $t$ test, and non-normally distributed data were analysed by the Wilcoxon signed-rank test. Data were presented as the median and 95\% confidence interval. For nominal outcomes, the difference was analysed by the marginal homogeneity test and Stuart-Maxwell test. Data were presented as numbers (percent). Binary logistic regression was used to describe the risk of getting PCMO and/ or VMT in elderly patients and patients with DM, using odds ratio (OR) and 95\% confidence interval. Association testing between dry AMD, ACD, dense cataract, corneal haze, DM, and decreased

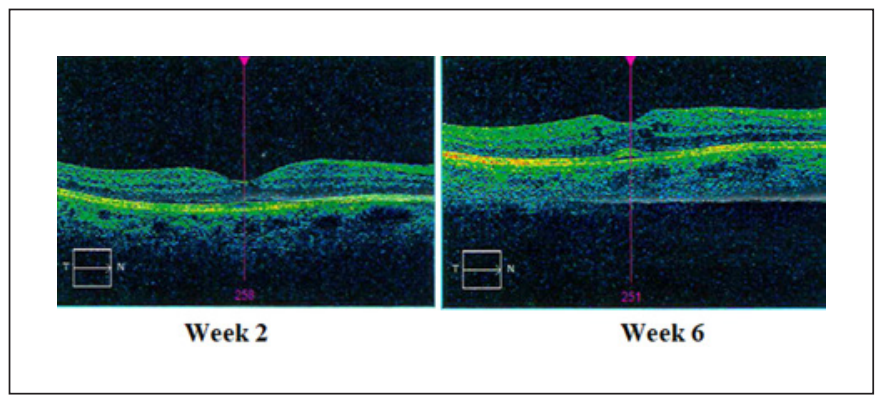

Fig. 1. Retinal OCT 6 weeks following cataract surgery illustrating macula with perifoveal cysts and localized foveal oedema indicative of PCMO.

visual acuity was performed using Pearson's $\chi^{2}$ test. Due to the low number of POAG, AMD, and young people ( $<65$ years old) $(n=4$, $n=5$, and $n=4$, respectively), the incidence of PCMO in these groups was not analysed.

\section{Results}

Ninety-two patients were initially recruited for the study. Seven patients withdrew over the course of the study, with a final total of 85 patients (58.8\% female; 72.8 years average age; and $58.8 \%$ right eyes) who included and attended all appointments (Table 1). Eighty-one patients $(95 \%)$ were older than 65 years.

\section{Outcomes}

\section{Incidence of PCMO}

Overall PCMO was diagnosed in $8.2 \%$ of eyes at 2 weeks, $9.4 \%$ at 4 weeks, and $10.6 \%$ at 6 weeks post-operatively. When excluding risk factors for PCMO (VMT, DM, AMD, POAG, corneal haze, and dense cataract), the true incidence of PCMO was $6.3 \%, 6.3 \%$, and $4.2 \%$ at weeks 2,4 , and 6 post-operatively, respectively.

\section{Central Subfield Thickness}

Preoperative CST was $259 \mu \mathrm{m}$, and post-operative CST was $261 \mu \mathrm{m}$ at 2 weeks, $264 \mu \mathrm{m}$ at 4 weeks, and $263 \mu \mathrm{m}$ at 6 weeks (Table 2; Fig. 2a). CST was significantly increased at weeks $4(p=0.002)$ and $6(p<0.0001)$ compared to preoperative values.

\section{Intraocular Pressure}

Preoperative IOP was $16 \mathrm{~mm} \mathrm{Hg}, 15 \mathrm{~mm} \mathrm{Hg}$ at week 2, $13 \mathrm{~mm} \mathrm{Hg}$ at week 4, and $13 \mathrm{~mm} \mathrm{Hg}$ at week 6 postoperatively (Table 2; Fig. 2b). There was a significant de- 
Table 1. Demographics of our patient cohort $(n=85)$

\begin{tabular}{lll}
\hline Variable & Cataract patients, \% & \\
\hline Sex & & \\
$\quad$ Male & $35(41.2)$ & \\
$\quad$ Female & $50(58.8)$ & \\
Age & $72.8 \pm 8.146$ & \\
$\begin{array}{l}\text { Eye operated } \\
\text { Right }\end{array}$ & $50(58.8)$ & Without risk factors \\
$\quad$ Left & $35(41.2)$ & cohort $(n=47), \%$ \\
\hline & With risk factors, ${ }^{*}$ & $3(6.3)$ \\
& $\%$ & $3(6.3)$ \\
\hline PCMO incidence & & $2(4.2)$ \\
$\quad$ Week 2 post-operative & $7(8.2)$ & \\
$\quad$ Week 4 post-operative & $8(9.4)$ & \\
$\quad$ Week 6 post-operative & $9(10.6)$ & \\
DM & $20(23.5)$ & \\
VMT & $5(5.9)$ & \\
$\quad$ Preoperative & $13(15.3)$ & \\
Post-operative at week 2, 4 and 6 & $4(4.7)$ & \\
POAG & $5(5.9)$ & \\
AMD & $1(1.8)$ & \\
Corneal haze & $1(1.8)$ & \\
Dense cataract & & \\
\hline
\end{tabular}

Values for categorical variables are presented as $n(\%)$, values for continuous variables as mean \pm standard deviation. ${ }^{*}$ Risk factors $=$ VMT, DM, POAG, AMD, corneal haze, and dense cataracts.
Table 2. Post-operative outcomes of CST, IOP, pachymetry, and BVA following cataract surgery

\begin{tabular}{|c|c|c|c|}
\hline Outcome & $N$ & Median $(95 \% \mathrm{Cl})$ & $p$ value \\
\hline \multicolumn{4}{|l|}{$\mathrm{CST}, \mu \mathrm{m}^{\S}$} \\
\hline Pre-operative & 61 & $259(254.65,266.57)$ & \\
\hline Week 2 & 71 & $261(256.75,267.05)$ & 0.69 \\
\hline Week 4 & 67 & $264(258.26,270.37)$ & 0.002 \\
\hline Week 6 & 59 & $263(260.86,273.6)$ & $<0.0001$ \\
\hline \multicolumn{4}{|l|}{ IOP, mm Hg } \\
\hline Preoperative $^{\S}$ & 78 & $16(13.46,20.54)$ & \\
\hline Week 2 & 73 & $15(14.21,15.49)$ & $<0.0001$ \\
\hline Week 4 & 65 & $13(13.03,14.34)$ & $<0.0001$ \\
\hline Week 6 & 61 & $13(12.46,13.83)$ & $<0.0001$ \\
\hline \multicolumn{4}{|l|}{ Pachymetry, $\mu \mathrm{m}^{\S}$} \\
\hline Day 1 & 75 & $569(556.99,584.01)$ & \\
\hline Week 2 & 70 & $558(547.8,568.62)$ & $<0.001$ \\
\hline Week 4 & 63 & $551(543.63,564.7)$ & $<0.001$ \\
\hline Week 6 & 59 & $550.5(538.4,560.98)$ & $<0.001$ \\
\hline \multicolumn{4}{|l|}{ BCVA, logMAR } \\
\hline Week $2^{\S}$ & 74 & $-0.018(0.078)$ & \\
\hline Week 4 & 64 & $-0.038(0.073)$ & 0.018 \\
\hline Week 6 & 61 & $-0.047(0.066)$ & 0.024 \\
\hline
\end{tabular}

crease in IOP at all post-operative time points compared to preoperative levels $(p<0.0001)$.

\section{Pachymetry}

Preoperative central corneal thickness was $569 \mu \mathrm{m}$, and post-operative corneal thickness was $558 \mu \mathrm{m}$ at week 2, $551 \mu \mathrm{m}$ at week 4, and $550.5 \mu \mathrm{m}$ at week 6 (Table 2; Fig. 2c). There was a significant decrease in central corneal thickness at all post-operative time points compared to preoperative values $(p<0.001)$.

Visual Acuity

Seventy-two patients (84\%) achieved 6/18 (equivalent to $0.5 \log$ MAR) or better UDVA at day 1 post-operatively, 79 patients (93\%) at week 2 , and 82 patients $(96 \%)$ at week 6 post-operatively. Visual acuity remained stable in UDVA at all post-operative time points (weeks 2, 4, and $6)$ compared to BCVA week 2 post-operatively $(p<0.05)$. There was no statistically significant change in UDVA at week 6 post-operatively in comparison with BCVA weeks 2 and 4 post-operatively, indicating stable visual acuity.

Seventy-nine patients (93\%) achieved $6 / 6$ or better BCVA at week 2 post-operatively $(-0.018 \pm 0.078$ log- 


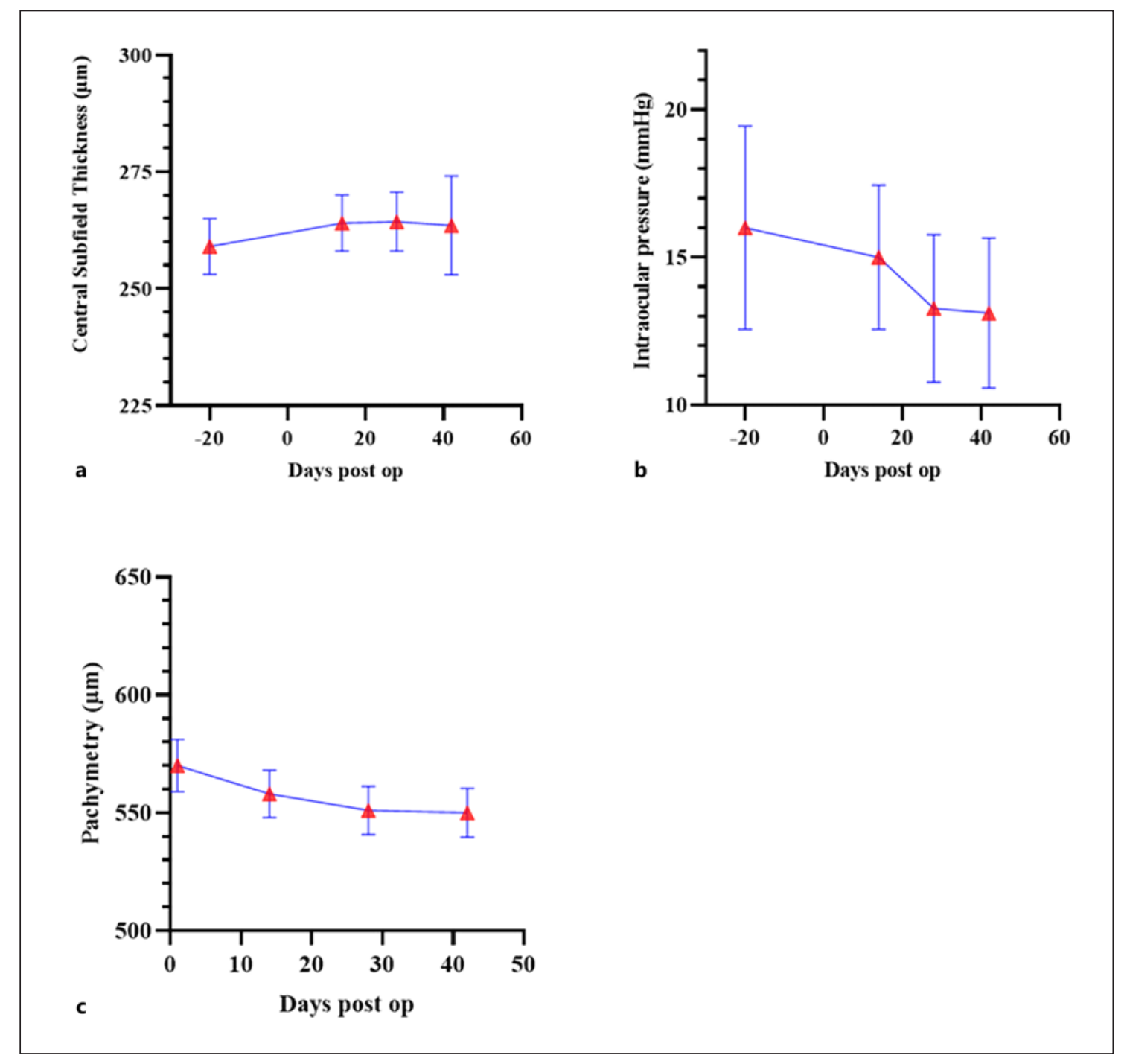

Fig. 2. a CST pre- and post-cataract surgery. b IOP pre- and post-cataract surgery. c Corneal thickness postcataract surgery.

MAR), 82 patients $(97 \%)$ at week $4(-0.038 \pm 0.073 \mathrm{Log}$ MAR) and 84 patients (99\%) at week $6(-0.047 \pm 0.066$ LogMAR) (Table 2; Fig. 3). There was significant visual stability in BCVA at all post-operative time points (weeks 4 and 6) compared to week 2 value $(p<0.05)$.

\section{Vitreomacular Traction}

A total of 18 patients had VMT in this study, 5 preoperatively (5.9\%) and $13(15.3 \%)$ at weeks 2,4 , and 6 postoperatively. Both preoperative and post-operative VMT were significantly correlated with PCMO $\left(\chi^{2}=5.15, p=\right.$ 0.023 and $\chi^{2}=6.68, p<0.0001$, respectively; Table 1 ).
Association between PCMO and VMT with Known Risk Factors

A total of 20 patients had DM in this study, and 20\% developed PCMO by week 6 post-operatively. PCMO and diabetic patients had a non-significant increase in the risk of PCMO at week $2(\mathrm{OR}=1.31, p=0.74)$, week $4(\mathrm{OR}=$ $2.12, p=0.34)$, and at week $6(\mathrm{OR}=3.0, p=0.13)$ postoperatively compared to the total sample ( $p=0.336$ and $p=0.13$, respectively; Table 3 ).

Patients with both preoperative VMT and DM had significant increased risk of PCMO at week $2(\mathrm{OR}=0.52$, $p=0.002)$, week $4(\mathrm{OR}=0.50, p=0.004)$, and week 6 postoperatively $(\mathrm{OR}=0.49, p=0.006)$, similar with patients 
Fig. 3. Snellen BCVA values at week 2, week 4, and week 6 post-cataract surgery.

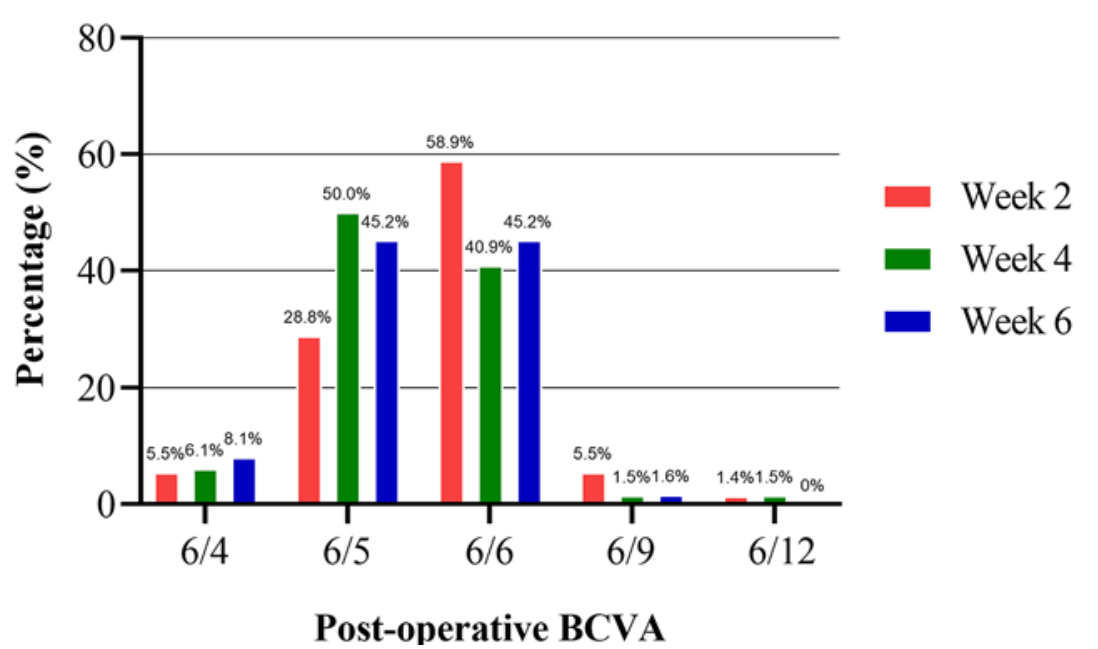

Table 3. Logistic regression between $\mathrm{PCMO}, \mathrm{VMT}$, and known risk factors

\begin{tabular}{|c|c|c|c|c|}
\hline & \multicolumn{2}{|l|}{$\mathrm{DM}^{*}$} & \multicolumn{2}{|l|}{$>65$ years old ${ }^{\S}$} \\
\hline & $\mathrm{Cl}$ range & $p$ value & $\mathrm{Cl}$ range & $p$ value \\
\hline \multicolumn{5}{|l|}{ PCMO } \\
\hline Preoperative & $1.09(0.11-11.08)$ & 0.943 & $0.529(0.051-5.523)$ & 0.595 \\
\hline Week 2 & $1.33(0.24-7.46)$ & 0.74 & $0.243(0.036-1.621)$ & 0.144 \\
\hline Week 4 & $2.12(0.46-9.77)$ & 0.336 & $0.602(0.11-3.28)$ & 0.557 \\
\hline Week 6 & $3(0.72-12.48)$ & 0.13 & $1.524(0.17-13.34)$ & 0.703 \\
\hline \multicolumn{5}{|l|}{$\mathrm{VMT}^{+}$} \\
\hline Preoperative & 15.7 (1.64-15.77) & 0.014 & 0 & 0 \\
\hline Post-operative & $3.22(0.864-12.01)$ & 0.083 & 0 & 0 \\
\hline
\end{tabular}

All data are presented as $\mathrm{OR}, 95 \% \mathrm{Cl}, p$ value. $\mathrm{Cl}$, confidence interval. * Reference (nondiabetics). ${ }^{\S}$ Reference ( $\leq 65$ years old). ${ }^{\dagger}$ Patients with both preoperative VMT and DM had significant increased risk of PCMO at week $2(\mathrm{OR}=0.52, p=0.002)$, week $4(\mathrm{OR}=0.50, p=$ $0.004)$, and week 6 post-operatively $(O R=0.49, p=0.006)$, similar with patients with postoperative VMT and DM at week $2(\mathrm{OR}=0.51, p=0.005)$, week $4(\mathrm{OR}=0.49, p=0.003)$, and week 6 post-operatively $(\mathrm{OR}=0.47, p=0.002)$. with post-operative VMT and DM at week $2(\mathrm{OR}=0.51$, $p=0.005)$, week $4(\mathrm{OR}=0.49, p=0.003)$, and week 6 postoperatively $(\mathrm{OR}=0.47, p=0.002)$. Patients over 65 years of age $(n=81)$ had a non-significant increase in risk of PCMO at week $2(\mathrm{OR}=0.529)$, week $4(\mathrm{OR}=0.557)$, and at week 6 post-operatively $(\mathrm{OR}=1.524)$ compared to the total sample $(p=0.557$ and $p=0.703$, respectively; Table 3). Lastly, a non-significant correlation was found between $\operatorname{ACD}\left(\chi^{2}=7.64, p=0.17\right)$, dense cataract $\left(\chi^{2}=\right.$ $3.75, p=0.585)$, corneal haze $\left(\chi^{2}=3.75, p=0.585\right)$, DM $\left(\chi^{2}=3.54, p=0.618\right)$, and decreased visual acuity at week 6 post-operatively.

\section{Discussion}

This study investigated the incidence of PCMO in patients who underwent cataract surgery at Shellharbour Public Hospital in NSW, Australia. CST increased throughout the follow-up period and peaked at 4-6 weeks post-operatively. IOP steadily decreased as visual acuity remained stable post-operatively. Diabetes and increased age ( $>65$ years) were associated with an increased risk of developing PCMO; however, the data were not statistically significant. 
This study reports an overall incidence of PCMO of $8.2 \%$ which increased to $10.6 \%$ by 6 weeks post-operatively. Despite this figure, after excluding at-risk populations, the true incidence was $4.2 \%$ by week 6 which was mainly offset by excluding patients with diabetes, of which $20 \%$ developed PCMO. Our true incidence is slightly higher than other reported rates of PCMO, which range from $0.1 \%$ to $2.35 \%$, but our overall incidence is consistent with studies that assessed the incidence of PCMO in at risk groups [13, 17, 21, 22]. A large cohort study by Chu et al. [16] reported $1.17 \%$ of non-diabetic patients developed PCMO, increasing to $10.63 \%$ in diabetic, pan-retinal photocoagulation patients. Do et al. [23] reported a $25 \%$ incidence of PCMO in diabetic groups, and Schimier et al. [15] also reported an increased risk of PCMO due to DM $(\mathrm{RR}=1.77)[15,23]$. Diabetes creates a pro-inflammatory ocular environment, whereby cytokines reduce blood flow of choriocapillaris, a mechanism which may predispose DM patients to PCMO [24]. These findings are therefore consistent and agree with previous research that patients with diabetes are at a greater risk of developing PCMO following cataract surgery [25-29].

While our findings are supported by similar incidence rates in other studies, differences in testing, diagnosis protocols, and image acquisition amongst studies may confound the generalizability of results. Currently, there is no global consensus for PCMO diagnostic criteria postcataract surgery which is problematic for establishing true incidence and leads to high variability in design, diagnostic methods, and surgical techniques between studies [30]. In our cohort, tests were performed by trained clinical staff, and a diagnosis of PCMO was determined by the surgeon according to the following criteria: CST $>295 \mu \mathrm{m}$ and perifoveal cysts and localized foveal oedema (Fig. 1) [31]. Direct comparisons between studies can be difficult as diagnosis criteria are not always reported. Some reported methods from other PCMO studies have used OCT and fluorescein angiography $[27,32]$, yellowish discolouration of intra-retinal and perifoveal cystoid spaces [33], and OCT angiography for non-invasive visualization of the retinal vasculature [27, 34-37]. These varied approaches to diagnosis may have impacts on perceived incidence between cohorts and may explain the differing rates of PCMO between studies.

Recent evidence reveals that VMT leads to macular oedema through myofibroblast differentiation, leading to glycation and collagen crosslinking $[38,39]$. In our cohort, patients with pre- and post-operative VMT were associated with PCMO, and patients with both DM and pre- and post-operative VMT were at a greater risk of

Post-Cataract Cystoid Macular Oedema developing PCMO. Post-operatively acquired VMT in diabetes patients was positively correlated but not significant $(p=0.08)$. Diabetic retinopathy is a known risk factor for developing VMT, and so, diabetic patients with preoperative VMT may have more advanced diabetes, in turn predisposing them to macular oedema. The patients that did not develop VMT despite having diabetes may have not experienced the same degree of physiological changes as those having VMT preoperatively, and our sample size may have exacerbated this effect. Bhagat et al. [40] reported a 10- to 20-fold increase in glycation levels in diabetic vitreous than non-diabetics. A wide range of studies observed a positive effect of pars plana vitrectomy on diabetic macular oedema, VMT, and retinal thickening. Ikeda et al. [41] reported that $28 \%-49 \%$ of patients who underwent vitrectomy had improved visual outcomes, cystoid changes disappeared in 5 days, and macular oedema improved within 2 weeks. Despite these known associations, our study showed no significant association between post-operative VMT and DM (OR = 2.917) which may be explained by our small sample size and exclusion of post-vitrectomy patients. Nevertheless, data suggest that patients with DM who have not undergone vitrectomy may not be at increased risk of developing VMT following cataract surgery.

Visual acuity was stable by 6 weeks post-cataract surgery in our data sample, with no significant change in UCVA between weeks 2 and 6 despite high PCMO incidence (Table 2). On the other hand, Chu et al. [16] reported a significant decrease in VA from 0.567 preoperatively to 0.328 post-operatively. After recovery from macular oedema, visual acuity did not recover to $6 / 6$ in $26.8 \%$ of the Hunter et al. [42] cohort. A recent meta-analysis also reported a significant decrease in visual acuity in PCMO eyes [43]. This finding could be explained by the alteration of parafoveal and foveal retina structure, but the impact of post-operative visual acuity on PCMO incidence remains unclear [42]. Macular thickness at 6 weeks post operation should be observed by OCT and fluorescein angiography diagnostic tests in order to identify PCMO in a timely manner as late development of PCMO has been reported even after uncomplicated surgery [44].

For patients with DM, subconjunctival triamcinolone has been beneficial in the past for decreasing PCMO, but considering $20 \%$ of our DM cohort still developed PCMO, it had a little clinical benefit in our cohort [45]. Given the high rates of $\mathrm{PCMO}$ generally in the DM populations, it is fair to conclude pharmaceutical management is suboptimal, and future efforts should be directed towards de- 
veloping more evidence-based medical therapies. For patients with VMT and DM before undergoing cataract surgery, it may be beneficial to undergo a vitrectomy to reduce the chances of developing post-operative PCMO or delaying cataract surgery for spontaneous resolution of VMT which has been observed in $11 \%$ of cases within 15 months $[46,47]$.

Our post-operative management may have played a role in our reported PCMO incidence as the benefit of different treatment regimens was unclear at the time of the study, especially prophylaxis [48-50]. A decisive 2021 prospective RCT trial has determined that a preoperative 3-day nepafenac regime does significantly reduce inflammation after cataract surgery [51], and in our study, no prophylaxis was used. Recent trials found sub-tenon triamcinolone and nepafenac, and intravitreal dexamethasone implantation reduced PCMO incidence $[52,53]$. In our study, we used a combination of ciprofloxacin $(0.3 \%$ $\mathrm{w} / \mathrm{v})$, diclofenac sodium $(0.1 \% \mathrm{w} / \mathrm{v})$, and phenylephrine hydrochloride $(1 \% \mathrm{w} / \mathrm{v}) /$ prednisolone acetate $(0.12 \%$ $\mathrm{w} / \mathrm{v}$ ) eye drops post-operatively, which at the time was aligned with the best practice. More recently, nepafenac has shown beneficial effects over diclofenac, ketorolac tromethamine, and bromfenac and should be considered a more effective NSAID in post-operative care [51, 54]. Drops were discontinued 4 weeks after surgery, and our true incidence of PCMO dropped by $2.1 \%$ between 4 and 6 weeks post-operatively. This suggests that pharmacological management was well timed and reduced inflammation at critical time points for this cohort. It has been observed by a Cochrane review that PCMO typically peaks at 4 weeks post-operatively; however, we showed a consistent incidence of PCMO between 2 and 4 weeks before dropping at 6 , which may have been observed due to small sample size [50]. Larger cohort studies should seek to define the most vulnerable time points for developing PCMO in healthy non-comorbid populations to ensure that pharmacological management is temporally administered to combat the critical time points for PCMO.

Our study has several strong points; we selected highly reported risk factors and analysed their contribution to PCMO incidence. We measured data on day 1 and weeks 2,4 , and 6 post-cataract surgery to observe any significant changes. Despite this, our limitations include a small cohort size which may lead to type 2 errors. Furthermore, patients were recruited at day 1 post-operatively, meaning that data from the immediate preoperative period are not available for comparison. This may skew pre- and post-operative comparisons. No qualitative data were compiled to evaluate patient satisfaction and perception, which would provide information about the impact of PCMO on surgery satisfaction.

\section{Conclusion}

Overall, the incidence of PCMO was highest in patients with comorbidities, especially diabetes. DM and advanced age were highly associated with PCMO, and there was no evidence of increased risk with prostaglandin use. As the true incidence of PCMO was found to be greatest at 2-4 weeks post-operatively, we support the immediate post-operative treatment regime of NSAIDs. The consensus on treatment regimens for the comorbid populations, especially DM patients, remains limited and we recommend future efforts targeting better pharmaceutical management for these groups.

\section{Acknowledgements}

The authors acknowledge all of Wollongong Eye Specialists staff for their help. The authors also acknowledge and thank Dr. Alvin Tan and Dr. Inca Hutchison for obtaining research ethics and assisting in data collection for this project.

\section{Statement of Ethics}

This study protocol was reviewed and approved by the University of Wollongong/ISLHD Health and Medical Human Research Ethics Committee, approval number 2016/008. Informed written consent was obtained from participants (or their parent/legal guardian/next of kin) to participate in the study. The patients gave their written informed consent to publish the data (including publication of images).

\section{Conflict of Interest Statement}

The authors have no conflicts of interest to disclose.

\section{Funding Sources}

This research did not receive any specific grant from funding agencies in the public, commercial, or not-for-profit sector. The authors have no financial relationships relevant to this article to disclose. 


\section{Author Contributions}

I.S. contributed to methodology, investigation, project administration, formal data analysis, writing - original draft, and writing - review and editing. G.B. contributed to formal data analysis, writing - original draft, and writing - review and editing. A.T. contributed to methodology, investigation, project administration, formal data collection, and formal data analysis. E.T. contributed to formal data collection, methodology, and writing - review and editing. S.A. contributed to conceptualization, methodology, supervision, writing - review, and editing.

\section{Data Availability Statement}

Further enquiries can be directed to the corresponding author.

\section{References}

1 Tasman W, Jaeger EA. Duane's foundations of clinical ophthalmology. Philadelphia: Lippincott Williams \& Wilkins; 1996.

2 Khanna R, Pujari S, Sangwan V. Cataract surgery in developing countries. Curr Opin Ophthalmol. 2011 Jan;22(1):10-4.

3 Riaz Y, de Silva SR, Evans JR. Manual small incision cataract surgery (MSICS) with posterior chamber intraocular lens versus phacoemulsification with posterior chamber intraocular lens for age related cataract. Cochrane Database Syst Rev. 2013(10):CD008813.

4 Ugarte M. Pseudophakic cystoid macular oedema. Cataract surgery. Berlin: Springer; 2021. p. 173-89.

5 Irvine SR. A newly defined vitreous syndrome following cataract surgery. Am J Ophthalmol. 1953 May;36(5):599-619.

6 Bradford JD, Wilkinson CP, Bradford RH Jr. Cystoid macular edema following extracapsular cataract extraction and posterior chamber intraocular lens implantation. Retina. 1988;8(3):161-4

7 Schubert HD. Cystoid macular edema: the apparent role of mechanical factors. Prog Clin Biol Res. 1989;312:277-91.

8 Hudes GR, Li WY, Rockey JH, White P. Prostacyclin is the major prostaglandin synthesized by bovine retinal capillary pericytes in culture. Invest Ophthalmol Vis Sci. 1988 29(10):1511-6.

9 Jonas JB, Kreissig I, Degenring RF. Intravitreal triamcinolone acetonide for pseudophakic cystoid macular edema. Am J Ophthalmol. 2003;136(2):384-6.

10 Flach AJ. The incidence, pathogenesis and treatment of cystoid macular edema following cataract surgery. Trans Am Ophthalmol Soc. 1998;96:557.

11 Packer M, Lowe J, Fine H. Incidence of acute postoperative cystoid macular edema in clinical practice. J Cataract Refract Surg. 2012 Dec; 38(12):2108-11.

12 Chu CJ, Johnston RL, Buscombe C, Sallam $\mathrm{AB}$, Mohamed Q, Yang YC. Risk factors and incidence of macular edema after cataract surgery: a database study of 81,984 eyes. Ophthalmology. $2016 \mathrm{Feb} ; 123(2): 316-23$.

13 Henderson BA, Kim JY, Ament CS, FerrufinoPonce ZK, Grabowska A, Cremers SL. Clinical pseudophakic cystoid macular edema: Risk factors for development and duration after treatment. J Cat Refract Surg. 2007;33(9):1550-8.
14 Guo S, Patel S, Baumrind B, Johnson K, Levinsohn D, Marcus E, et al. Management of pseudophakic cystoid macular edema. Survey Ophthalmol. 2015;60(2):123-37.

15 Schmier JK, Halpern MT, Covert DW, Matthews GP. Evaluation of costs for cystoid macular edema among patients after cataract surgery. Retina. 2007;27(5):621-8.

16 Chu CJ, Johnston RL, Buscombe C, Sallam $\mathrm{AB}$, Mohamed Q, Yang YC, et al. Risk factors and incidence of macular edema after cataract surgery: a database study of 81984 eyes. Ophthalmology. 2016;123(2):316-23.

17 McCafferty S, Harris A, Kew C, Kassm T, Lane L, Levine J, et al. Pseudophakic cystoid macular edema prevention and risk factors; prospective study with adjunctive once daily topical nepafenac $0.3 \%$ versus placebo. BMC Ophthalmol. 2017 Feb 20;17(1):16.

18 Erikitola OO, Siempis T, Foot B, Lockington $D$. The incidence and management of persistent cystoid macular oedema following uncomplicated cataract surgery: a Scottish ophthalmological surveillance unit study. Eye. 2021 Feb;35(2):584-91.

19 McNamara P, Hutchinson I, Thornell E, Batterham M, Iloski V, Agarwal S. Refractive stability following uncomplicated cataract surgery. Clin Exp Optom. 2019 Mar;102(2):154-

20 Duker JS, Kaiser PK, Binder S, de Smet MD, Gaudric A, Reichel E, et al. The international vitreomacular traction study group classification of vitreomacular adhesion, traction, and macular hole. Ophthalmology. 2013;120(12): 2611-9.

21 Rossetti L, Autelitano A. Cystoid macular edema following cataract surgery. Curr Opin Ophthalmol. 2000;11(1):65-72.

22 Bélair M-L, Kim SJ, Thorne JE, Dunn JP, Kedhar SR, Brown DM, et al. Incidence of cystoid macular edema after cataract surgery in patients with and without uveitis using optical coherence tomography. Am J Ophthalmol. 2009;148(1):128-35.

23 Do JR, Oh JH, Chuck RS, Park CY. Transient corneal edema is a predictive factor for pseudophakic cystoid macular edema after uncomplicated cataract surgery. Korean J Ophthalmol. 2015;29(1):14-22.
24 Pierru A, Carles M, Gastaud P, Baillif S. Measurement of subfoveal choroidal thickness after cataract surgery in enhanced depth imaging optical coherence tomography. Invest Ophthalmol Vis Sci. 2014;55(8):4967-74.

25 Khurana RN, Palmer JD, Porco TC, Wieland MR. Dexamethasone intravitreal implant for pseudophakic cystoid macular edema in patients with diabetes. Ophthalmic Surg Lasers Imaging Retina. 2015;46(1):56-61.

26 Munk MR, Jampol LM, Simader C, Huf W, Mittermüller TJ, Jaffe GJ, et al. Differentiation of diabetic macular edema from pseudophakic cystoid macular edema by spectral-domain optical coherence tomography. Investigative Ophthalmol Visual Sci. 2015;56(11): 6724-33.

27 Yang J, Cai L, Sun Z, Ye H, Fan Q, Zhang K, et al. Risk factors for and diagnosis of pseudophakic cystoid macular edema after cataract surgery in diabetic patients. J Cataract Refract Surg. 2017;43(2):207-14.

28 Ylinen P, Laine I, Lindholm JM, Tuuminen R. Poor glycemic control as a risk factor for pseudophakic cystoid macular edema in patients with diabetes. J Cataract Refract Surg. 2017;43(11):1376-82.

29 Yüksel B, Karti Ö, Kusbeci T. Topical nepafenac for prevention of post-cataract surgery macular edema in diabetic patients: patient selection and perspectives. Clin Ophthalmol. 2017;11:2183.

30 Han JV, Patel DV, Squirrell D, McGhee CN. Cystoid macular oedema following cataract surgery: a review. Clin Exp Ophthalmol. 2019; 47(3):346-56.

31 Cirrus HD-OCT macular thickness analysis report. Jena: Carl Zeiss Meditec; 2011.

32 Lobo C. Pseudophakic cystoid macular edema. Ophthalmologica. 2012;227(2):61-7.

33 Rossetti L, Autelitano A. Cystoid macular edema following cataract surgery. Curr Opin Ophthalmol. 2000 Feb;11(1):65-72.

34 Frost NA, Sparrow JM, Strong NP, Rosenthal AR. Vitreous loss in planned extracapsular cataract extraction does lead to a poorer visual outcome. Eye. 1995;9(Pt 4):446-51.

35 Arcieri ES, Santana A, Rocha FN, Guapo GL, Costa VP. Blood-aqueous barrier changes after the use of prostaglandin analogues in patients with pseudophakia and aphakia: a 6-month randomized trial. Arch Ophthalmol. 2005 Feb;123(2):186-92. 
36 Sacconi R, Corbelli E, Carnevali A, Mercuri S, Rabiolo A, Querques L, et al. Optical coherence tomography angiography in pseudophakic cystoid macular oedema compared to diabetic macular oedema: qualitative and quantitative evaluation of retinal vasculature. $\mathrm{Br} \mathrm{J}$ Ophthalmol. 2018 Dec;102(12):1684-90.

37 Sacconi R, Corbelli E, Carnevali A, Mercuri S, Rabiolo A, Querques L, et al. Optical coherence tomography angiography in pseudophakic cystoid macular oedema compared to diabetic macular oedema: qualitative and quantitative evaluation of retinal vasculature. $\mathrm{Br} \mathrm{J}$ Ophthalmol. 2018;102(12):1684-90.

38 Gandorfer A, Rohleder M, Kampik A. Epiretinal pathology of vitreomacular traction syndrome. Br J Ophthalmol. 2002;86(8):902-9.

39 Figueroa MS, Contreras I, Noval S. Surgical and anatomical outcomes of pars plana vitrectomy for diffuse nontractional diabetic macular edema. Retina. 2008 Mar;28(3):4206.

40 Bhagat N, Grigorian RA, Tutela A, Zarbin MA. Diabetic macular edema: pathogenesis and treatment. Surv Ophthalmol. 2009;54(1): 1-32.

41 Ikeda T, Sato K, Katano T, Hayashi Y. Vitrectomy for cystoid macular oedema with attached posterior hyaloid membrane in patients with diabetes. Br J Ophthalmol. 1999; 83(1):12-4.

42 Hunter AA, Modjtahedi SP, Long K, Zawadzki R, Chin EK, Caspar JJ, et al. Improving visual outcomes by preserving outer retina morphology in eyes with resolved pseudophakic cystoid macular edema. J Cataract Refract Surg. 2014;40(4):626-31.
43 Kessel L, Tendal B, Jørgensen KJ, Erngaard D, Flesner P, Andresen JL, et al. Post-cataract prevention of inflammation and macular edema by steroid and nonsteroidal anti-inflammatory eye drops: a systematic review. Ophthalmology. 2014;121(10):1915-24.

44 Cagini C, Fiore T, Iaccheri B, Piccinelli F, Ricci MA, Fruttini D. Macular thickness measured by optical coherence tomography in a healthy population before and after uncomplicated cataract phacoemulsification surgery. Curr Eye Res. 2009;34(12):1036-41.

45 Wielders LHP, Schouten JSAG, Winkens B, Van Den Biggelaar FJHM, Veldhuizen CA, Findl O, et al. European multicenter trial of the prevention of cystoid macular edema after cataract surgery in nondiabetics: ESCRS PREMED study report 1. J Cataract Refract Surg. 2018;44(4):429-39.

46 Selver OB, Parlak M, Soylemezoglu ZO, Saatci AO. Spontaneous resolution of vitreomacular traction: a case series. Clin Exp Optom. 2013;96(4):424-7.

47 Petrou P, Chalkiadaki E, Errera M-H, Liyanage S, Wickham L, Papakonstantinou E, et al. Factors associated with the clinical course of vitreomacular traction. J Ophthalmol. 2020;2020:9457670.

48 Kim SJ, Schoenberger SD, Thorne JE, Ehlers JP, Yeh S, Bakri SJ. Topical nonsteroidal antiinflammatory drugs and cataract surgery: a report by the American academy of ophthalmology. Ophthalmology. 2015; 122(11): 2159-68.
49 Lim BX, Lim CH, Lim DK, Evans JR, Bunce C, Wormald R. Prophylactic non-steroidal anti-inflammatory drugs for the prevention of macular oedema after cataract surgery. Cochrane Database Syst Rev. 2016;11(11): CD006683.

50 Juthani VV, Clearfield E, Chuck RS. Non-steroidal anti-inflammatory drugs versus corticosteroids for controlling inflammation after uncomplicated cataract surgery. Cochrane Database Syst Rev. 2017;7(7):CD010516.

51 Cagini C, Cerquaglia A, Pellegrino A, Iannone A, Lupidi M, Fiore T. Effect of preoperative topical nepafenac $0.1 \%$ on inflammatory response after uncomplicated cataract surgery in healthy subjects. Acta Ophthalmol. 2021;99(1):e70-3.

52 Bellocq D, Pierre-Kahn V, Matonti F, Burillon C, Voirin N, Dot C, et al. Effectiveness and safety of dexamethasone implants for postsurgical macular oedema including IrvineGass syndrome: the EPISODIC-2 study. Br J Ophthalmol. 2017;101(3):333-41.

53 Yüksel B, Uzunel UD, Kerci SG, Sağban L, Küsbeci T, Örsel T. Comparison of subtenon triamcinolone acetonide injection with topical nepafenac for the treatment of pseudophakic cystoid macular edema. Ocular Immunol Inflamm. 2017;25(4):513-9.

54 Sahu S, Ram J, Bansal R, Pandav SS, Gupta A. Effect of topical ketorolac $0.4 \%$, nepafenac $0.1 \%$, and bromfenac $0.09 \%$ on postoperative inflammation using laser flare photometry in patients having phacoemulsification. J Cataract Refract Surg. 2015;41(10):2043-8. 